\title{
Endoreversible Thermodynamics: A Tool for Simulating and Comparing Processes of Discrete Systems ${ }^{\ddagger}$
}

\author{
Wolfgang Muschik ${ }^{1, *}$ and Karl Heinz Hoffmann ${ }^{2}$ \\ 1 Institut für Theoretische Physik, Technische Universität Berlin, 10623 Berlin, \\ Germany \\ 2 Institut für Physik, Technische Universität Chemnitz, 09107 Chemnitz, Germany \\ *Corresponding author (muschik@physik.tu-berlin.de)
}

\begin{abstract}
Endoreversible thermodynamics is concerned with reversible sub-systems that are in irreversible interaction with each other. Consequently, endoreversible thermodynamics represents the analogue for discrete systems to the local equilibrium hypothesis in continuum thermodynamics. Here a real cyclic 2reservoir process is simulated by different endoreversible model processes. Simulation means that the simulating process has the same net heat exchanges, cycle time, power, entropy production, and efficiency as the original one. By introducing process-independent simulation parameters as constraints for the irreversible interaction, a family of comparative endoreversible processes is generated including the simulation of the original process. This procedure allows the process parameters of the family of comparative processes to be compared to those of the original one. The fraction "power of the real process over the maximal power inbetween the comparative family" is introduced as a parameter describing the process excellence.
\end{abstract}

\section{Introduction}

If a cyclic process is described by thermostatics, no real time appears. Thus reversible (timeless) cyclic processes, such as the Carnot, the Joule, the Otto and the Diesel processes [2], are idealized, quasi-processes in the equilibrium

\footnotetext{
$\$$ Partly delivered at the 9 th International Symposium on Continuum Models and Discrete Systems [1]. 
subspace that do not exist in nature [3]. Nevertheless, the efficiencies of these "processes" are often used for comparing them with efficiencies of real running processes. This procedure seems questionable; we ask whether there are more realistic processes for comparing them with real running machines. It is exactly this question which stood at the beginning of the development of finite time thermodynamics. Since then, a lot of progress has been made in general non-equilibrium thermodynamics. In this paper, we turn again to the original question and study the problem of finding these more realistic expressions for the efficiency of irreversible heat to power conversion processes. We will use dissipative, but still idealized processes as described in a special thermodynamic discipline, called endoreversible thermodynamics, which was developed in the last 20 years [4-8].

An endoreversible system is defined by a set of reversible sub-systems that interact with each other irreversibly. Of course, an endoreversible machine is still not a real running one, because it includes reversible sub-systems. But because irreversibility is modeled by the dissipative interaction of these reversible subsystems, such an endoreversible machine may provide better "processes" for comparing them with those of a real running machine than pure reversible "processes". For the special case of 2-reservoir processes, we investigate how far this program of achieving more realistic estimations of efficiencies and other thermodynamic performance criteria of real running machines by endoreversible ones can be carried out.

The aim of the paper is mostly conceptual. While heat engines with several heat sources show interesting results [9, 10], cyclic 2-reservoir processes are the most simple systems to discuss the concepts involved. For this purpose, we recall in the second section the real cyclic 2-reservoir processes, and we discuss the connections between entropy production, efficiency, power, and work. In the third section, we define what a simulation and what a model of a real running process is. The concept of a simulation of a real process (a simulating process) is introduced in order to elucidate the difference to a model of the real running machine. While a simulating process by definition has the same thermodynamic exchanges as the real one under consideration, it is unsuitable for comparison. Therefore, a family of comparative processes is introduced by fixing the simulation parameters after having chosen a special model for the real running machine. In the following sections, we investigate different endoreversible models for 2-reservoir processes in closed systems. These models are the Carnot engine with a heat leak [11], the Novikov engine with two different kinds of heat conduction, and the Novikov engine with heat leak [12, 13], which has recently proven its ability in matching even the behavior of highfeatured engines [14]. 


\section{Real cyclic 2-reservoir processes}

We consider two heat reservoirs $(\mathrm{H}$ and $\mathrm{L})$ of different thermostatic (equilibrium) temperatures $T_{\mathrm{H}}>T_{\mathrm{L}}$. A real, cyclic, irreversible 2-reservoir process is operating between these two reservoirs exchanging the heat flows $\dot{Q}_{\mathrm{H}}(t)$ and $\dot{Q}_{\mathrm{L}}(t)$ with $\mathrm{H}$ and $\mathrm{L}$, respectively. No mass and no work exchange appear between the reservoirs and the system undergoing the cyclic process. The heat exchanges during a cycle of the cycle time $\tau>0$ are

$$
Q_{\mathrm{H}}:=\int_{0}^{\tau} \dot{Q}_{\mathrm{H}}(t) d t, \quad Q_{\mathrm{L}}:=\int_{0}^{\tau} \dot{Q}_{\mathrm{L}}(t) d t .
$$

Throughout the paper, we will consider heat to work conversion processes that are characterized by a negative work $W \leq 0$, i.e., the system delivers work to the environment during a cycle. Therefore, the First Law for heatpower processes states

$$
Q_{\mathrm{H}}+Q_{\mathrm{L}}+W=0, \quad W \leq 0, \Rightarrow Q_{\mathrm{H}} \geq-Q_{\mathrm{L}}>0 .
$$

A thermodynamic diagram of a 2-reservoir cyclic heat-power process is shown in Figure 1.

The entropy production per cycle $\Sigma$ appearing in the system is given by the entropy exchanges between the system and its controlling reservoirs $\mathrm{H}$ and $\mathrm{L}$ :

$$
\Sigma:=-\frac{Q_{\mathrm{H}}}{T_{\mathrm{H}}}-\frac{Q_{\mathrm{L}}}{T_{\mathrm{L}}} \geq 0
$$

According to the Second Law, the entropy production $\Sigma$ is not negative. This inequality represents the special form of Clausius's inequality for 2-reservoir systems [15]. From Eqs. (2) and (3) we obtain

$$
1 \leq \alpha:=-\frac{Q_{\mathrm{H}}}{Q_{\mathrm{L}}} \leq \frac{T_{\mathrm{H}}}{T_{\mathrm{L}}} \rightarrow Q_{\mathrm{H}}=-\alpha Q_{\mathrm{L}} .
$$

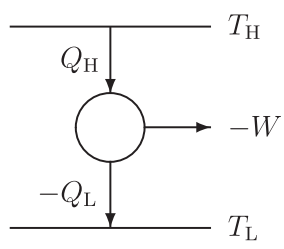

Figure 1 Thermodynamic diagram of a 2-reservoir cyclic heat-power process. 


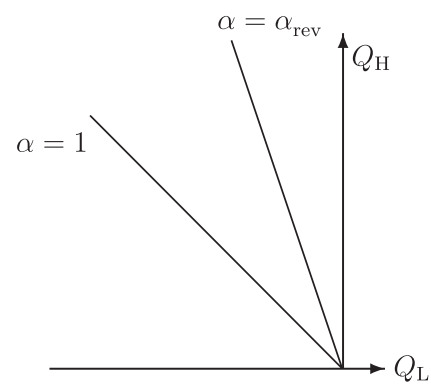

Figure 2 Power-producing thermodynamic cyclic processes are located between $\alpha=1$ and $\alpha=\alpha_{\text {rev }}$. Different values of $\alpha$ characterize different cyclic processes.

These inequalities demonstrate that all existing processes possess an $\alpha$ that is restricted by the Second Law and that is determined by $Q_{H}$ and $Q_{L}$. The equality in Eq. (3) is valid for reversible processes, for which Eq. (4) results in

$$
\alpha_{\mathrm{rev}}=\frac{T_{\mathrm{H}}}{T_{\mathrm{L}}} \geq \alpha
$$

According to Eqs. (4) and (5), all power-producing thermodynamic cyclic processes have an $\alpha$ that is located in the angle between $\alpha=1$ and $\alpha=\alpha_{\text {rev }}$ in Figure 2. For real processes with non-vanishing heat exchanges, it is known that $\alpha_{\text {rev }}$ can only be approached if $\tau \rightarrow \infty$, i.e., if the cycle time of the process becomes arbitrarily long. That means that reversible processes are idealized, not-running "processes" that do not exist in nature.

The efficiency of a 2-reservoir process is defined by [16]

$$
0 \leq \eta(\alpha):=\frac{-W}{Q_{\mathrm{H}}}=1+\frac{Q_{\mathrm{L}}}{Q_{\mathrm{H}}}=1-\frac{1}{\alpha} \leq 1-\frac{T_{\mathrm{L}}}{T_{\mathrm{H}}}=: \eta_{\mathrm{rev}},
$$

and its power by

$$
P:=\frac{-W}{\tau}=Q_{\mathrm{H}} \frac{\eta}{\tau}=P\left(Q_{\mathrm{H}}, \eta, \tau\right) \geq 0 .
$$

Inserting Eqs. (6) and (4), the power results in

$$
P\left(Q_{\mathrm{L}}, \alpha, \tau\right)=\frac{Q_{\mathrm{H}}}{\tau}\left(1-\frac{1}{\alpha}\right)=\frac{Q_{\mathrm{L}}}{\tau}(1-\alpha)
$$

For the reversible limit $\tau \rightarrow \infty$, we obtain

$$
P_{\text {rev }}=0 .
$$


The entropy production (3) per cycle becomes by Eqs. (3), (4), and (6)

$$
\Sigma\left(Q_{\mathrm{H}}, \alpha\right)=Q_{\mathrm{H}}\left(\frac{1}{\alpha T_{\mathrm{L}}}-\frac{1}{T_{\mathrm{H}}}\right)=\frac{Q_{\mathrm{H}}}{T_{\mathrm{L}}}\left(\eta_{\mathrm{rev}}-\eta\right)=\Sigma\left(Q_{\mathrm{H}}, \eta\right) \geq 0,
$$

which yields for the reversible limit $\Sigma_{\mathrm{rev}}=0$.

Using Eq. (10) to eliminate $\eta$ in Eq. (7), we obtain for the power of the process

$$
P=\frac{1}{\tau}\left(Q_{\mathrm{H}} \eta_{\mathrm{rev}}-\Sigma T_{\mathrm{L}}\right)=\frac{-W}{\tau}=\frac{\Sigma T_{\mathrm{L}}}{\tau}\left(\frac{Q_{\mathrm{H}} \eta_{\mathrm{rev}}}{\Sigma T_{\mathrm{L}}}-1\right) .
$$

By use of Eq. (10) and later on of Eq. (6), this results in

$$
P=\frac{\Sigma T_{\mathrm{L}}}{\tau} \frac{\eta}{\eta_{\mathrm{rev}}-\eta}=\frac{\Sigma T_{\mathrm{H}}}{\tau} \frac{\alpha-1}{\alpha_{\mathrm{rev}}-\alpha}
$$

The expressions for the entropy production, power, and efficiency derived here will be needed later on for simulating real cyclic 2-reservoir processes. For a more systematic elucidation, we introduce some formal tools in the next section.

\section{Machines, process class, and family}

As Figure 1 shows, all real cyclic 2-reservoir processes can be described by five quantities:

$$
\boldsymbol{z}:=\left(T_{\mathrm{H}}, T_{\mathrm{L}}, Q_{\mathrm{H}}, Q_{\mathrm{L}}, \tau\right) \in \mathscr{M}^{5},
$$

which span a 5-dimensional manifold. The physical meaning of the parameters spanning the manifold induces some restrictions: $T_{H}>T_{L}>0, Q_{H} \geq$ $-Q_{L}>0$, satisfying Eq. (4) and $\tau>0$. For arbitrary but fixed allowed values of the quantities $\left(T_{\mathrm{H}}, T_{\mathrm{L}}, Q_{\mathrm{H}}, Q_{\mathrm{L}}, \tau\right)$, we call $\boldsymbol{z}$ a process class and $\mathscr{M}^{5}$ the set of all process classes. According to its definition, the process class contains all processes having the same values for the reservoir temperatures, the heat exchanges, and the cycle time. That means that we concern ourselves no longer with the particular time dependence of the heat flows during the cyclic process, but we group together all processes having the same net exchanges to form the process class. Concerning the net exchanges (1), all processes of a process class are equivalent to each other. 
Later on, we have to compare different process classes belonging to the same controlling heat reservoirs. Therefore we define the process family,

$$
\mathscr{M}^{3}\left(T_{\mathrm{H}}, T_{\mathrm{L}}\right)=\left(T_{\mathrm{H}}, T_{\mathrm{L}}, *, *, *\right),
$$

as a 3-dimensional manifold for fixed $T_{\mathrm{H}}$ and $T_{\mathrm{L}}$. According to Eqs. (4) and (6), each member of the process family can be described by different but equivalent sets of variables:

$$
(*, *, *) \rightarrow\left(\hat{Q}_{\mathrm{H}}, \hat{Q}_{\mathrm{L}}, \hat{\tau}\right),\left(\hat{Q}_{\mathrm{H}}, \hat{\alpha}, \hat{\tau}\right),\left(\hat{Q}_{\mathrm{L}}, \hat{\alpha}, \hat{\tau}\right),\left(\hat{Q}_{\mathrm{L}}, \hat{\eta}, \hat{\tau}\right) .
$$

Here ^ denotes a variable in contrast to the arbitrary but fixed values of $Q_{\mathrm{H}}$, $Q_{\mathrm{L}}, \tau$ of the process class (13). Note that also other parameterizations in Eq. (15) are possible using the entropy production or the power.

Each process class belongs to the process family (14)

$$
\left(T_{\mathrm{H}}, T_{\mathrm{L}}, Q_{\mathrm{H}}, Q_{\mathrm{L}}, \tau\right) \in \mathscr{M}^{3}\left(T_{\mathrm{H}}, T_{\mathrm{L}}\right),
$$

but there are members of the process family that do not belong to the process class because of $\hat{Q}_{\mathrm{H}} \neq Q_{\mathrm{H}}, \hat{Q}_{\mathrm{L}} \neq Q_{\mathrm{L}}$ and $\hat{\tau} \neq \tau$. Of course, the process family (14) is a subset of the set of all process classes $\mathscr{M}^{5}$ in Eq. (13).

Real power-producing heat engine cycles are performed by machines. Examples are the many existing internal combustion engines or the turbines in power plants. Usually these machines can operate between a variety of bath temperatures and at different speeds. Here we restrict ourselves to the process family (14) defined by arbitrary but fixed family parameters $\left(T_{\mathrm{H}}, T_{\mathrm{L}}\right)$. Then a given machine will not be able to realize all possible members of the process family. In general, two different 2-reservoir machines $(I$ and $I I)$ performing processes of equal cycle time and equal absorbed heat,

$$
\tau^{I}=\tau^{I I} \quad \text { and } \quad Q_{\mathrm{H}}^{I}=Q_{\mathrm{H}}^{I I}, \quad \text { but } \quad Q_{\mathrm{L}}^{I} \neq Q_{\mathrm{L}}^{I I},
$$

may have different efficiencies and consequently different heat exchanges at $T_{\mathrm{L}}$. Therefore, we need for the description of special machines "constitutive equations", which couple variables of the process family to each other, thus decreasing the dimension of $\mathscr{M}^{3}$. This dependence of the variables on each other for special machines is called a machine diagram,

$$
Q_{\mathrm{L}}=\Omega\left(Q_{\mathrm{H}}, \tau\right), \quad \text { or } \quad \eta=\Xi\left(Q_{\mathrm{H}}, \tau\right), \quad \alpha=\Psi\left(Q_{\mathrm{L}}, \tau\right) \text {, etc. }
$$

describing individual properties of the special machine. By the machine diagram, a 2-dimensional process subfamily is introduced, because one of the 


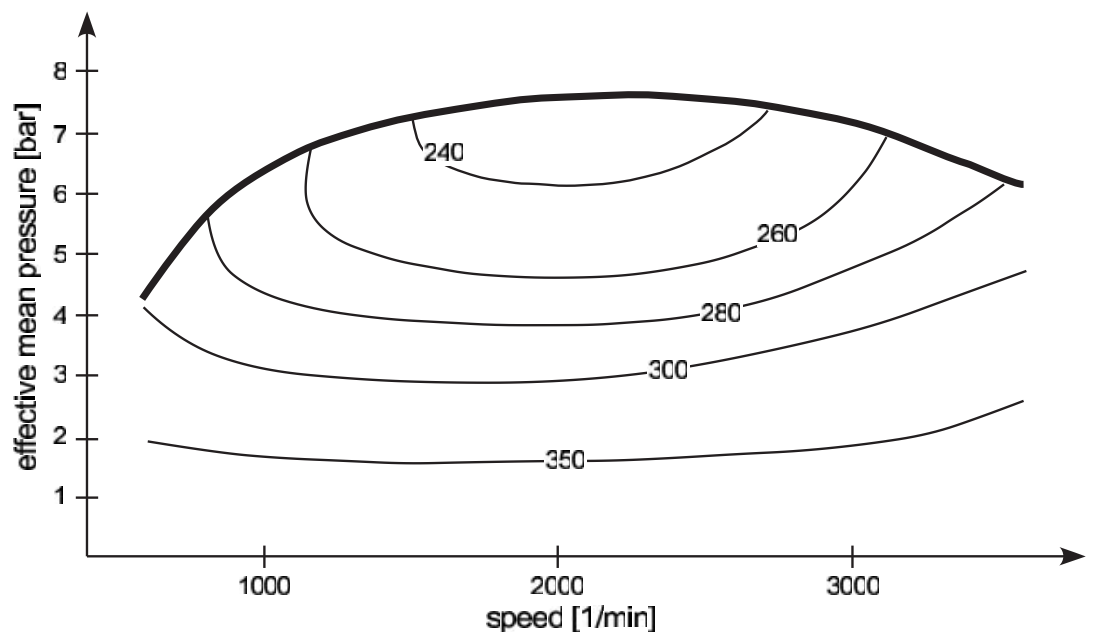

Figure 3 Machine diagram for a 4-cylinder engine showing the lines of equal fuel consumption.

three variables of the process family (14) is determined by Eq. (18). Machine diagrams are, e.g., fuel consumption maps of a 4-cylinder engine (Figure 3).

As Figure 3 shows, the constitutive mappings $\Omega, \Xi, \Psi$, etc., belonging to the 2-reservoir machine are not unique in general: they are divided into branches $\Omega_{k}, \Xi_{l}, \Psi_{m}$ (k, l, m are the numbers of the branches): for a fixed cycle time $\tau$ and fixed fuel supply $Q_{\mathrm{H}}$, there are two possible values for the effective mean pressure (which is proportional to the work/power) in Figure 3. Consequently, also two possible values for $Q_{\mathrm{L}}, \alpha$, and $\eta$ follow. That means, for fixed cycle time and fixed fuel supply, the engine can work in two different process classes,

$$
\left(T_{\mathrm{H}}, T_{\mathrm{L}}, Q_{\mathrm{H}}, Q_{\mathrm{L}}^{I}, \tau\right) \neq\left(T_{\mathrm{H}}, T_{\mathrm{L}}, Q_{\mathrm{H}}, Q_{\mathrm{L}}^{I I}, \tau\right)
$$

with different efficiencies.

According to Eq. (18), for special machines Eqs. (7) and (10) result in

$$
P_{l}\left(Q_{\mathrm{H}}, \tau\right)=\frac{Q_{\mathrm{H}}}{\tau} \Xi_{l}\left(Q_{\mathrm{H}}, \tau\right), \quad \Sigma_{l}\left(Q_{\mathrm{H}}, \tau\right)=\frac{Q_{\mathrm{H}}}{T_{\mathrm{L}}}\left[\eta_{\mathrm{rev}}-\Xi_{l}\left(Q_{\mathrm{H}}, \tau\right)\right]
$$

depending on the branch of the constitutive mapping. We will omit this branch index in the sequel.

Note that $P$ and $\Sigma$ attain their extrema for different values $\left(Q_{\mathrm{H}}^{*}, \tau^{*}\right) \neq$ $\left(Q_{\mathrm{H}}^{\diamond}, \tau^{\diamond}\right)$ of the family variables $Q_{\mathrm{H}}$ and $\tau$ : 


$$
P^{\mathrm{extr}}=\frac{Q_{\mathrm{H}}^{*}}{\tau^{*}} \Xi\left(Q_{\mathrm{H}}^{*}, \tau^{*}\right), \quad \Sigma^{\mathrm{extr}}=\frac{Q_{\mathrm{H}}^{\diamond}}{T_{\mathrm{L}}}\left[\eta_{\mathrm{rev}}-\Xi\left(Q_{\mathrm{H}}^{\diamond}, \tau^{\diamond}\right)\right] .
$$

This result is easy to understand: If the entropy production $\Sigma$ is minimal, that is according to Eq. (10) in case of a reversible process, the work $-W$ is maximal according to Eq. (11),

$$
\eta=\eta_{\mathrm{rev}} \Rightarrow \Sigma \rightarrow \text { minimal } \Rightarrow-W=Q_{\mathrm{rev}} \eta_{\mathrm{rev}}-\Sigma T_{\mathrm{L}} \rightarrow \text { maximal },
$$

but the power $P$ is zero due to $\tau \rightarrow \infty$. Consequently, if $P$ is maximal for a special cycle time $\tau, \Sigma$ does not have an extremal value for this process. Because according to Eq. (10), the efficiency $\eta$ is monotonously decreasing with increasing entropy production; the statement "The higher the efficiency, the greater the power" is wrong: efficiency is a process quantity that is defined independently of the cycle time, whereas the power is not. Therefore, efficiency and cycle time are independent variables of the process family according to Eq. (15). But for each special machine, the efficiency depends experimentally on $\tau$ according to the machine diagram (18) and Figure 3.

If a real running machine characterized by its machine diagram (18) is investigated, the question arises how "good" is this machine with regard to its efficiency. To answer this question, one has to compare this machine with efficiencies of other ones. But how to choose these comparing machines? Because we do not know any specific internal structure of the 2-reservoir machines considered in this section, we will first simulate them all by endoreversible ones whose internal structures are known and which are models simulating the original machine. Then we can embed each of the endoreversible models into a specially constructed set of comparing machines whose efficiencies can be compared with that of the original one, thus answering the question of its quality.

\section{Simulation and model}

Now we want to simulate a process class of real cyclic 2-reservoir processes described by Eq. (13). That means we have to replace the original cyclic process by a special other process. Of course, this replacement is not unique: there are many other processes simulating the original one. "Simulating" means that the process replacing the original one has the same reservoir temperatures, the same heat exchanges, and the same cycle time as the original process: simulating process and original process belong to the same process class $z$.

For simulating, here we use processes of endoreversible systems. While endoreversible systems can be of nearly arbitrary complexity $[6,7]$, we confine 
ourselves in this paper to endoreversible systems that are a combination of reversible Carnot engines combined with irreversible transport laws such as the Fourier or the Newton law of heat conduction.

We are now able to define what the endoreversible simulation of a process means:

Definition: Processes of endoreversible systems that have the same heat exchanges, the same cycle time, the same work and power, the same entropy production and efficiency as the original process are called simulating processes.

Endoreversible simulating processes can be performed by differently composed endoreversible machines. This gives rise to the following definition:

Definition: A model of a process class is a specially constructed machine undergoing a simulating process with respect to the process class in consideration induced by the real running original process.

By these two definitions, the distinction between simulation and model becomes clear: a simulation of the original process cannot be distinguished from it by considering the exterior, because the simulating process is in the same process class (13) as the original one. There are a lot of simulating processes, all belonging to the same process class. These simulating processes can be modelled totally different. Here we are using endoreversible models, but other modelling is possible, e.g., by reversible processes.

According to Eqs. (13) and (14), each process class belongs to a process family. But a process family is larger than the process class belonging to it. Consequently, the process class is embedded in the process family, which therefore contains more processes than the simulating ones. This embedding of the simulating processes into the process family makes it possible to compare the original process (and all simulating processes) with special members of the family that are not simulating it. We will define and discuss these comparative processes in Section 6.

In the next section, we will consider several endoreversible systems, and we will show how to construct simulation parameters for the later introduction of comparative processes.

\section{Endoreversible systems}

Endoreversible machines consist of reversible subsystems that interact irreversibly with each other [5-7]. This irreversible interaction of the subsystems 
is the source of entropy production. Because endoreversible machines contain by definition reversible subsystems, they are - as the reversible machines idealized devices not existing in nature. In this section, we consider some examples of endoreversible machines for their later use as comparative models.

\subsection{Carnot engine with Fourier heat leak}

The entropy production (3) can be identically transformed into

$$
\Sigma=-\frac{Q_{\mathrm{H}}-\Delta Q}{T_{\mathrm{H}}}-\frac{Q_{\mathrm{L}}+\Delta Q}{T_{\mathrm{L}}}+\Delta Q\left(\frac{1}{T_{\mathrm{L}}}-\frac{1}{T_{\mathrm{H}}}\right)
$$

Note that the introduced heat exchange $\Delta Q$ is an arbitrary one without influencing the entropy production, the family parameters (14), and the heat exchanges (1) of the process family. The cycle time will be introduced later.

For constructing a particular endoreversible model, we choose $\Delta Q$ in such a way that the sum of the first two terms on the right-hand side of Eq. (22) become zero:

$$
-\frac{Q_{\mathrm{H}}-\Delta Q}{T_{\mathrm{H}}}-\frac{Q_{\mathrm{L}}+\Delta Q}{T_{\mathrm{L}}} \doteq 0
$$

We call Eq. (23) the reversibility condition because it represents Clausius's equality of a reversible process having the heat exchanges $Q_{\mathrm{H}}-\Delta Q$ and $Q_{\mathrm{L}}+\Delta Q$ between the system and the two controlling reservoirs of the temperatures $T_{\mathrm{H}}$ and $T_{\mathrm{L}}$, respectively (see Figure 4 ).

Taking Eq. (23) into account Eq. (22) results in

$$
\Sigma=\Delta Q\left(\frac{1}{T_{\mathrm{L}}}-\frac{1}{T_{\mathrm{H}}}\right)=-\frac{Q_{\mathrm{H}}}{T_{\mathrm{H}}}-\frac{Q_{\mathrm{L}}}{T_{\mathrm{L}}} \geq 0 \Rightarrow \Delta Q \geq 0 .
$$

The reversibility condition (23) determines the heat leak, $\Delta Q$, which is connected to the entropy production by Eqs. (24), (10), (4)2, and (5):

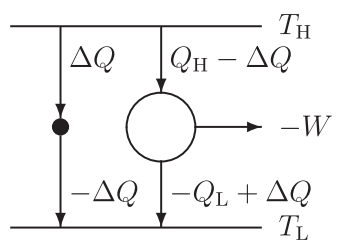

Figure 4 Thermodynamic diagram of a 2-reservoir cyclic process with heat leak. 


$$
\Delta Q=-Q_{\mathrm{L}}\left(\frac{1}{T_{\mathrm{L}}}-\frac{\alpha}{T_{\mathrm{H}}}\right) /\left(\frac{1}{T_{\mathrm{L}}}-\frac{1}{T_{\mathrm{H}}}\right)=\frac{T_{\mathrm{H}} \Sigma\left(Q_{\mathrm{L}}, \alpha\right)}{\alpha_{\mathrm{rev}}-1} .
$$

The heat leak $\Delta Q$ is called a first simulation parameter. The endoreversible model of a real 2-reservoir process class is determined by specializing this first simulation parameter, which is given by the reversibility condition involving Eq. (23) or Eq. (25).

We now introduce a second simulation parameter $\lambda^{\text {hl }}$ by a "constitutive equation" for the heat leak including the cycle time of the original process class (13):

$$
\Delta Q=: \lambda^{\mathrm{hl}} \tau\left(\frac{1}{T_{\mathrm{L}}}-\frac{1}{T_{\mathrm{H}}}\right) \Rightarrow \lambda^{\mathrm{hl}}\left(Q_{\mathrm{L}}, \alpha, \tau\right) \geq 0 .
$$

This equation looks like a Fourier heat conduction ansatz, but it is not, because Eq. (26) determines the "heat conductivity" $\lambda^{\text {hl }}$, which of course is not a constant, but a function of $Q_{\mathrm{L}}, \alpha$, and $\tau$ according to Eqs. (26) and (25). From Eq. (26) follows by Eqs. (25) and (10)

$$
\lambda^{\mathrm{hl}}\left(Q_{\mathrm{L}}, \alpha, \tau\right)=\frac{\Sigma\left(Q_{\mathrm{L}}, \alpha\right)}{\tau\left(\frac{1}{T_{\mathrm{L}}}-\frac{1}{T_{\mathrm{H}}}\right)^{2}}=\frac{-Q_{\mathrm{L}} T_{\mathrm{H}}\left(\alpha_{\mathrm{rev}}-\alpha\right)}{\tau\left(\alpha_{\mathrm{rev}}-1\right)^{2}} .
$$

According to Eq. (10), we obtain from Eq. (27) in the reversible case

$$
\tau \lambda^{\mathrm{hl}}\left(Q_{\mathrm{L}}, \alpha_{\mathrm{rev}}, \tau\right)=0
$$

although $\tau \rightarrow \infty$ is valid for reversible processes. From Eq. (26) follows in this case

$$
\Delta Q_{\mathrm{rev}}=\lambda_{\mathrm{rev}}^{\mathrm{hl}}=0 .
$$

Consequently, as expected, there is no heat leak in the reversible case.

Replacing $Q_{\mathrm{L}} / \tau$ by Eq. $(27)_{2}$ in Eq. (8) we obtain for the power

$$
P\left(\alpha, \lambda^{\mathrm{hl}}\right)=\lambda^{\mathrm{hl}} \frac{1}{T_{\mathrm{H}}} \frac{\left(\alpha_{\mathrm{rev}}-1\right)^{2}}{\alpha_{\mathrm{rev}}-\alpha}(\alpha-1) .
$$

Now the original 2-reservoir process is simulated by an endoreversible model consisting of the reversible part described by Eq. (23) (the right-hand part of the diagram in Figure 4), and of an irreversible heat-conducting part, the heat 
leak (the left-hand part of the diagram in Figure 4), described by Eq. (24). The endoreversible model undergoes the same process as the original one; both belong to the same process class.

If we now change the original real running process, say by changing the cycle time, then the altered process is still in the process family (14), but no longer in the process class (13). Additionally, the original process is performed by a special machine for which its machine diagram (18) is valid (Figure 3). Consequently, the three variables in Eq. (15) are not independent of each other according to Eq. (18), and $\alpha$ in Eq. (30) has to be replaced by its values $\Psi\left(Q_{\mathrm{L}}, \tau\right)$ given by the machine diagram.

\subsection{Novikov engine with Fourier heat conduction}

We now simulate the original 2-reservoir process family by using another endoreversible model, the Novikov engine [12] (see Figure 5).

The entropy production (3) is now identically transformed into

$$
\Sigma=-\frac{Q_{\mathrm{H}}}{T_{\mathrm{H}}}-\frac{Q_{\mathrm{L}}}{T_{\mathrm{L}}}=Q_{\mathrm{H}}\left(\frac{1}{T_{\mathrm{i}}}-\frac{1}{T_{\mathrm{H}}}\right)-\frac{Q_{\mathrm{H}}}{T_{\mathrm{i}}}-\frac{Q_{\mathrm{L}}}{T_{\mathrm{L}}}
$$

by introducing $T_{\mathrm{i}}$ as the first simulation parameter. The reversibility condition for the reversible part of the endoreversible Novikov process is chosen as

$$
-\frac{Q_{\mathrm{H}}}{T_{\mathrm{i}}}-\frac{Q_{\mathrm{L}}}{T_{\mathrm{L}}} \doteq 0
$$

which leads to the entropy production

$$
\Sigma=Q_{\mathrm{H}}\left(\frac{1}{T_{\mathrm{i}}}-\frac{1}{T_{\mathrm{H}}}\right)
$$

The first simulation parameter is thus fixed by Eq. (32):

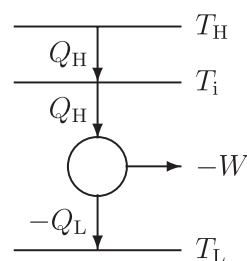

Figure 5 Thermodynamic diagram of the Novikov process.

J. Non-Equilib. Thermodyn. $\cdot 2006 \cdot$ Vol. $31 \cdot$ No. 3 


$$
1 \leq \alpha:=-\frac{Q_{\mathrm{H}}}{Q_{\mathrm{L}}}=\frac{T_{\mathrm{i}}}{T_{\mathrm{L}}} \Rightarrow T_{\mathrm{i}} \geq T_{\mathrm{L}}, T_{\mathrm{i}}=\alpha T_{\mathrm{L}}
$$

The second simulation parameter $\lambda^{\mathrm{np}}$ is introduced in the same fashion as in Eq. (26) and is determined by a "constitutive equation":

$$
\begin{aligned}
& Q_{\mathrm{H}}=\lambda^{\mathrm{np}} \tau\left(\frac{1}{T_{\mathrm{i}}}-\frac{1}{T_{\mathrm{H}}}\right)=-\alpha Q_{\mathrm{L}}, \\
& \lambda^{\mathrm{np}}\left(Q_{\mathrm{L}}, \alpha, \tau\right)=\left(-\alpha Q_{\mathrm{L}}\right) / \tau\left(\frac{1}{\alpha T_{\mathrm{L}}}-\frac{1}{T_{\mathrm{H}}}\right) .
\end{aligned}
$$

By Eq. (35), the entropy production (33) results in

$$
\Sigma=\lambda^{\mathrm{np}} \tau\left(\frac{1}{T_{\mathrm{i}}}-\frac{1}{T_{\mathrm{H}}}\right)^{2},
$$

which is analogous to Eq. $(27)_{1}$. The power (8) becomes by use of Eqs. (35) and $(34)_{3}$

$$
P\left(\alpha, \lambda^{\mathrm{np}}\right)=\lambda^{\mathrm{np}}\left(1-\frac{1}{\alpha}\right)\left(\frac{1}{\alpha T_{\mathrm{L}}}-\frac{1}{T_{\mathrm{H}}}\right) .
$$

This expression is of course different from Eq. (30) due to the different models for the original process, although the values of the power in Eqs. (30) and (38) are equal to that of the original process in Eq. (7). For a special original machine, $\alpha$ is given by Eq. (18) 3 . From Eq. (37) we obtain Eq. (9) again.

Of course, it is equally possible to use $T_{\mathrm{i}}$ as the first simulation parameter instead of $\alpha$. Then from Eq. (8) the power $P$ becomes

$$
P\left(T_{\mathrm{i}}, Q_{\mathrm{H}}\right)=\frac{Q_{\mathrm{L}}}{\tau}(1-\alpha)=\frac{-Q_{\mathrm{H}}}{\alpha \tau}(1-\alpha)=\frac{Q_{\mathrm{H}}}{\tau}\left(1-\frac{T_{\mathrm{L}}}{T_{\mathrm{i}}}\right) .
$$

Using Eq. (35), we finally obtain

$$
P\left(T_{\mathrm{i}}, \lambda^{\mathrm{np}}\right)=\frac{\lambda^{\mathrm{np}}}{T_{\mathrm{H}}}\left(1-\frac{T_{\mathrm{L}}}{T_{\mathrm{i}}}\right)\left(\frac{T_{\mathrm{H}}}{T_{\mathrm{i}}}-1\right) .
$$

The same result could have been directly obtained from Eq. (38), if $\alpha$ were replaced by $T_{\mathrm{i}} / T_{\mathrm{L}}$. 


\subsection{Novikov engine with Newtonian heat conduction}

We now simulate the original 2-reservoir process again by the Novikov process [12] (see Figure 5), but this time we use a different simulation for the "heat conduction", the second simulation parameter. Instead of Eq. (35), we introduce a different second simulation parameter $\lambda^{\mathrm{N}}$ by requiring

$$
Q_{\mathrm{H}}=\lambda^{\mathrm{N}} \tau\left(T_{\mathrm{H}}-T_{\mathrm{i}}\right) \Rightarrow \lambda^{\mathrm{N}}=\frac{Q_{\mathrm{H}}}{\tau\left(T_{\mathrm{H}}-\alpha T_{\mathrm{L}}\right)} .
$$

In this case, the expression for the power (39) becomes

$$
P\left(T_{\mathrm{i}}, \lambda^{\mathrm{N}}\right)=\lambda^{\mathrm{N}} T_{\mathrm{L}}\left(1-\frac{T_{\mathrm{i}}}{T_{\mathrm{L}}}\right)\left(1-\frac{T_{\mathrm{H}}}{T_{\mathrm{i}}}\right) .
$$

Note that the process class (13) can still be described by the new simulation parameters $\lambda^{\mathrm{N}}$ and $T_{\mathrm{i}}$ or $\alpha$. Inserting the proper values (41) and (34) for $\lambda^{\mathrm{N}}$ and $T_{\mathrm{i}}$ still results in the original value for the power.

\subsection{Novikov engine with Fourier heat leak}

Another endoreversible model is the Novikov engine with heat leak (see Figure 6). For the process performed by this engine, the decomposition of the entropy production (3) by introducing two first simulation parameters $\Delta Q$ and $T_{\mathrm{i}}$ states

$$
\Sigma=Q_{\mathrm{H}}\left(\frac{1}{T_{\mathrm{i}}}-\frac{1}{T_{\mathrm{H}}}\right)+\Delta Q\left(\frac{1}{T_{\mathrm{L}}}-\frac{1}{T_{\mathrm{i}}}\right)-\frac{Q_{\mathrm{H}}-\Delta Q}{T_{\mathrm{i}}}-\frac{Q_{\mathrm{L}}+\Delta Q}{T_{\mathrm{L}}} .
$$

In contrast to the preceding simulations, only one of these parameters is determined by the reversibility condition:

$$
-\frac{Q_{\mathrm{H}}-\Delta Q}{T_{\mathrm{i}}}-\frac{Q_{\mathrm{L}}+\Delta Q}{T_{\mathrm{L}}}=0,
$$

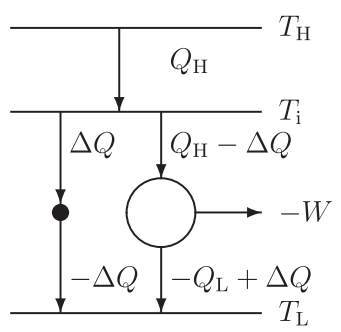

Figure 6 Thermodynamic diagram of a 2-reservoir Novikov engine with heat leak. 
which results in a relation between the two first simulation parameters $\Delta Q$ and $T_{\mathrm{i}}$,

$$
\Delta Q=-Q_{\mathrm{L}}\left(\frac{\alpha}{T_{\mathrm{i}}}-\frac{1}{T_{\mathrm{L}}}\right) /\left(\frac{1}{T_{\mathrm{i}}}-\frac{1}{T_{\mathrm{L}}}\right) .
$$

The entropy production is according to Eqs. (43) and (44):

$$
\Sigma=Q_{\mathrm{H}}\left(\frac{1}{T_{\mathrm{i}}}-\frac{1}{T_{\mathrm{H}}}\right)+\Delta Q\left(\frac{1}{T_{\mathrm{L}}}-\frac{1}{T_{\mathrm{i}}}\right) .
$$

Hence the power (12) becomes

$$
P=\frac{1}{\tau}\left[Q_{\mathrm{H}}\left(\frac{1}{T_{\mathrm{i}}}-\frac{1}{T_{\mathrm{H}}}\right)+\Delta Q\left(\frac{1}{T_{\mathrm{L}}}-\frac{1}{T_{\mathrm{i}}}\right)\right] \frac{\alpha-1}{\frac{1}{T_{\mathrm{L}}}-\frac{\alpha}{T_{\mathrm{H}}}} .
$$

Note that one of the first simulation parameters can be chosen arbitrarily because the reversibility condition (44) determines only one of them. If one of the two the parameters is chosen, the value of the other one is adjusted in such a way that entropy production and power are those of the original process.

Two second simulation parameters $\lambda$ and $\kappa$ are introduced by

$$
Q_{\mathrm{H}}=\lambda \tau\left(\frac{1}{T_{\mathrm{i}}}-\frac{1}{T_{\mathrm{H}}}\right), \quad \Delta Q=\kappa \tau\left(\frac{1}{T_{\mathrm{L}}}-\frac{1}{T_{\mathrm{i}}}\right) .
$$

Both the second simulation parameters depend on $T_{\mathrm{i}}, Q_{\mathrm{L}}, \alpha$, and $\tau$ :

$$
\lambda=\lambda\left(Q_{\mathrm{L}}, \alpha, \tau, T_{\mathrm{i}}\right), \quad \kappa=\kappa\left(Q_{\mathrm{L}}, \alpha, \tau, T_{\mathrm{i}}\right)
$$

By Eq. (48), the expression of the power (47) results in

$$
P\left(\alpha, \kappa, \lambda, T_{\mathrm{i}}\right)=\left[\lambda\left(\frac{1}{T_{\mathrm{i}}}-\frac{1}{T_{\mathrm{H}}}\right)^{2}+\kappa\left(\frac{1}{T_{\mathrm{L}}}-\frac{1}{T_{\mathrm{i}}}\right)^{2}\right] \frac{\alpha-1}{\frac{1}{T_{\mathrm{L}}}-\frac{\alpha}{T_{\mathrm{H}}}} .
$$

As already mentioned, $T_{\mathrm{i}}$ can be chosen arbitrarily because the combination of the quantities $\lambda, \kappa$, and $T_{\mathrm{i}}$ appearing in Eq. (50) has by construction the restricting property that $\lambda$ and $\kappa$ change with $T_{\mathrm{i}}$ in such a way that

$$
P\left(\alpha, \lambda, \kappa, T_{\mathrm{i}}\right)=P\left(Q_{\mathrm{L}}, \alpha, \tau\right)
$$

is independent of $T_{\mathrm{i}}$. 
In this section we discussed four endoreversible models of a general 2reservoir process family. Three of them, the process with heat leak and the two Novikov processes, are endowed with two simulation parameters, one of the first and one of the second kind, which are determined by the reversibility condition and by the "constitutive equation" for the heat exchange. The fourth model, the Novikov process with heat leak, has double the number of simulation parameters. Consequently, one of them can be chosen arbitrarily, because the reversibility condition and the two constitutive equations determine three simulation parameters. By construction - it is a simulation this free choice of one simulation parameter does not influence the entropy production and the power of the endoreversible model. How these models can be used for constructing comparative processes with respect to the real running process is discussed in the next section.

\section{Comparative processes}

In the last section, we considered simulations of the 2-reservoir process with different endoreversible models. The different expressions for the power (7), (11), (12), (30), (38), (42), and (50) are achieved by identical transformations, each conserving the value of the power of the original process. Thus all endoreversible simulations of the last section belong to the same original 2reservoir process; that means, all simulations and the original process are in the same process class.

We now raise the question again: "How efficient is the real 2-reservoir process?" The usual answer is that the real efficiency $\eta$ is compared with the reversible efficiency $\eta_{\text {rev }}$ in Eq. (6). This of course is a very simple procedure, because in this way a real, irreversible process is compared with an idealized, totally reversible one. In the light of the process family introduced at the beginning, one can interpret that comparison by stating that for all processes within the process family (14) one chooses the reversible Carnot process for a comparison and one chooses the efficiency as the criterion for that comparison. From the point of view of obtaining a "good" comparison process, this seems rather bold as all the many different processes (and remember, each is a whole process class) with different heat exchanges are lumped together. The reason for using the Carnot efficiency nonetheless is that one can be sure that the efficiencies of all the different members of the process family are bounded by the Carnot efficiency.

We will now discuss the question whether there are special processes, although they are also idealized and not running in nature, which are more suitable for comparison with real processes. Because the process class (13) is embedded in the appertaining process family (14), the idea arises to compare the efficiency 
of the original process (and therefore of the process class) with members of a specially constructed subfamily within the process family. The price one has to pay for a more suitable comparison will be the loss of the guaranteed inequality mentioned above. We will demonstrate that the subfamilies suggested will give different answers, and it remains an act of physical wisdom to choose a proper comparison family.

In the preceding section, we introduced a first and a second simulation parameter. The first one is defined by the reversibility condition, the second simulation parameter by the process class. Changing the process class will alter the second simulation parameter. We now choose an arbitrary but fixed process class (13) by which the value of the second simulation parameter, e.g., Eq. (26), is fixed. Then we embed the process class into a subfamily of the process family. The subfamily is generated by the restriction that all members of this subfamily possess the same value of the second simulation parameter of the embedded process class:

$$
\hat{\lambda}\left(\widehat{Q_{L}}, \hat{\alpha}, \hat{\tau}\right)=\lambda^{\circ}:=\lambda\left(Q_{L}, \alpha, \tau\right)=\text { const. }
$$

Here $\left(\widehat{Q_{L}}, \hat{\alpha}, \hat{\tau}\right)$ are variables of the process family according to Eq. (15), whereas $\left(Q_{L}, \alpha, \tau\right)$ are fixed by the process class. Now we can define the comparative processes as follows:

Definition: The subfamily $\left(T_{H}, T_{L}, \widehat{Q_{L}}, \hat{\alpha}, \hat{\tau}\right)$ generated by a constant second simulation parameter (52) induced by the chosen process class $\left(T_{H}, T_{L}, Q_{L}\right.$, $\alpha, \tau)$ is called the family of comparative processes.

We compare quantities that belong to the original process with the same quantities of the comparative family. But nothing is said about the effectiveness of this procedure, because for constructing the comparative family, here the "heat conductivities" are chosen to be constant (which is by no means strict, but is practiced by many people; see [6] for more details). This procedure shows the arbitrariness of finding a comparative family. But there is no other way to get rid of the reversible processes as comparative ones: simulation of the original process by models of the same process class and its extension to a process family for comparison.

A short remark of the concept of "model" is necessary: here the endoreversible models used in the preceding sections were used for simulating one special process class; that means, one special process of the original real running machine was simulated. Another process of the same machine belongs to another process class, was simulated by the same model, but with different simulation parameters. Because the values of the simulation parameters depend 
on the special process of the real running machine, the same models for simulation have different "constitutive properties".

But often the concept of "model" is used in another context: One model with fixed constitutive properties, here with fixed simulation parameters, should map all possible processes of the real running machine and not the only one in the process class. That means that the machine diagrams of the real running machine and that of the model should be identical, because otherwise the "model" is not a model. But most of the endoreversible models do not satisfy this additional restriction, as we easily can see: for the Novikov process with Fourier heat conduction (Section 5.2), we obtain from Eqs. (35) and (34):

$$
Q_{\mathrm{H}}=\lambda^{\circ} \tau\left(\frac{1}{\alpha T_{\mathrm{L}}}-\frac{1}{T_{\mathrm{H}}}\right),
$$

with a fixed value $\lambda^{\circ}$ for all possible processes of the real running machine. From this we get

$$
\frac{1}{\alpha}=\frac{Q_{H}}{\lambda^{\circ} \tau}+\frac{T_{L}}{T_{H}}
$$

and consequently, by Eq. (6),

$$
\eta=\eta_{\text {rev }}-\frac{Q_{H} T_{L}}{\lambda^{\circ} \tau}
$$

follows. All real machine diagrams have experimentally the property that there is a fuel supply $Q_{H}^{\dagger}$ and a cycle time $\tau^{\dagger}$ for which the efficiency is maximal. From Eq. (55), we obtain for constant $\lambda^{\circ}$

$$
\begin{aligned}
& \left.\frac{\partial \eta}{\partial \tau}\right|_{Q_{H}=Q_{H}^{\dagger}} ^{\tau=\tau^{\dagger}}=\frac{Q_{H}^{\dagger} T_{L}}{\lambda^{\circ} \tau^{\dagger 2}} \doteq 0, \\
& \left.\frac{\partial \eta}{\partial Q_{H}}\right|_{Q_{H}=Q_{H}^{\dagger}} ^{\tau=\tau^{\dagger}}=-\frac{T_{L}}{\lambda^{\circ} \tau^{\dagger}} \doteq 0 .
\end{aligned}
$$

The only solution is

$$
\left(\lambda^{\circ} \rightarrow \infty\right) \vee\left(\tau^{\dagger} \rightarrow \infty\right)
$$

describing the reversible limit according to Eq. (55) in which the power output vanishes according to Eqs. (38) or (39). Consequently, the model of a 
Novikov engine with Fourier heat conduction having the same constant "heat conductivities" for all possible process classes of the real running machine does not catch the reality, because it does not model the real machine diagram. The "heat conductivities" are only simulation parameters for constructing a comparative process family belonging to a single process class.

We now discuss the comparative processes for the simulations and models introduced above.

\subsection{Carnot engine with a Fourier heat leak}

The efficiency of a real process described by $Q_{\mathrm{H}}, Q_{\mathrm{L}}, \tau$ is to be compared to the efficiency of a Carnot engine with a Fourier heat leak. To be precise, we choose the adequate value for the heat conductance $\lambda^{\circ}$ by using Eq. (27) as a constraint for a process subfamily

$$
\lambda^{\circ} \tau\left(\frac{1}{T_{\mathrm{L}}}-\frac{1}{T_{\mathrm{H}}}\right)^{2}=-Q_{\mathrm{L}}\left(\frac{1}{T_{\mathrm{L}}}-\frac{\alpha}{T_{\mathrm{H}}}\right) .
$$

Then we consider the power (30) for the given constant value $\lambda^{\circ}$ according to Eq. (52):

$$
P\left(\alpha, \lambda^{\circ}\right)=\lambda^{\circ}\left(\frac{1}{T_{\mathrm{L}}}-\frac{1}{T_{\mathrm{H}}}\right)^{2}(\alpha-1) /\left(\frac{1}{T_{\mathrm{L}}}-\frac{\alpha}{T_{\mathrm{H}}}\right),
$$

and ask: "What is the process with the highest power in this subfamily?" This process is determined by varying $\alpha$ within its bounds for constant second simulation parameter $\lambda^{\circ}$ looking for the maximal power. Equation (60) shows that there is no internal extremum in $\alpha$. The power diverges as $\alpha$ approaches its boundary value $\alpha=T_{\mathrm{H}} / T_{\mathrm{L}}$, which represents the reversible case. This is physically unrealistic because of Eq. (59), which represents a machine diagram according to Eq. (18) 3 : In the reversible limit, Eq. (59) results in $\tau=0$ inducing the unlimited power. But we know that $\tau \rightarrow \infty$ is valid in the reversible case. Consequently, the machine diagram and therefore the Carnot engine with Fourier heat leak is not a suitable model for simulation. This will be different for the models discussed below.

\subsection{Novikov engine with Fourier heat conduction}

The same procedure we used in the last section is now applied to the Novikov model. The constraint for the comparative processes in that case is chosen by using the value of $\lambda^{\circ}$ from Eq. (36): 


$$
\lambda^{\circ} \tau\left(\frac{1}{\alpha T_{\mathrm{L}}}-\frac{1}{T_{\mathrm{H}}}\right)=-\alpha Q_{\mathrm{L}}
$$

This relation represents a machine diagram (18), reducing the number of independent variables in Eq. (15) to 2.

Now we have to look for the maximum of the power (38). An easy calculation results in

$$
\left.\frac{\partial P}{\partial \alpha}\right|_{\lambda}=0 \Rightarrow \alpha_{P_{\max }}=\frac{2}{1+\frac{T_{\mathrm{L}}}{T_{\mathrm{H}}}} .
$$

Note that due to the equivalence between $\alpha$ and $T_{\mathrm{i}}$, the equivalent calculation looking for the maximum of $P$ by varying $T_{\mathrm{i}}$ leads to the same result.

According to Eq. (6), we obtain for the efficiency belonging to the maximum power of the comparative Novikov process

$$
\eta_{\mathrm{N} . \mathrm{F}}=\frac{1}{2} \eta_{\mathrm{rev}}
$$

a result that is independent of the special value of $\lambda^{\circ}$ in Eq. (61). Because it also does not depend on the variables (15), this result is independent of the process class belonging to the original process. That means the comparative Novikov family characterized by Eq. (61) is a universal one, because of its independence of the special process class. The comparative processes in the Novikov model described by a fixed heat conductivity have their maximum power output for an efficiency (63) that is rather low. Again we stress that there are real processes running with a greater efficiency than $(1 / 2) \eta_{\text {rev }}$, but with less than maximal power output. Again we see that the efficiency is not a suitable measure for power output. Therefore we are looking for such a measure.

We now introduce the power comparison coefficient (PCC):

$$
\Pi(\eta):=\frac{P}{P^{\max }} .
$$

By Eq. (6), we obtain

$$
\begin{aligned}
& P\left(\alpha, \lambda^{n p}\right)=\lambda^{n p} \eta\left(\frac{1}{\alpha T_{L}}-\frac{1}{T_{H}}\right), \\
& \hat{P}^{\max }\left(\hat{\alpha}^{\text {Pmax }}, \lambda^{\circ}\right)=\lambda^{\circ} \hat{\eta}^{\text {Pmax }}\left(\frac{1}{\hat{\alpha}^{\text {Pmax }} T_{L}}-\frac{1}{T_{H}}\right), \quad \lambda^{n p} \doteq \lambda^{\circ} .
\end{aligned}
$$


Here $\hat{\eta}^{\text {Pmax }}$ is given by Eq. (63) and

$$
\frac{1}{\alpha T_{L}}-\frac{1}{T_{H}}=\frac{T_{H}-T_{L}-\eta T_{H}}{T_{L} T_{H}}=\frac{1}{T_{L}}\left(\eta^{r e v}-\eta\right)
$$

is valid. Inserting Eq. (63), this results in

$$
\frac{1}{\hat{\alpha}^{\text {Pmax }} T_{L}}-\frac{1}{T_{H}}=\frac{\left(T_{H}-T_{L}\right)}{2 T_{L} T_{H}}=\frac{\eta^{\text {rev }}}{2 T_{L}} .
$$

From Eqs. (65) and (66) follows after a short calculation

$$
\Pi(\eta)=4 \frac{\eta}{\eta^{r e v}}\left(1-\frac{\eta}{\eta^{r e v}}\right)
$$

A short calculation confirms the following inequalities for the PCC to hold:

$$
\begin{aligned}
& 0 \leq \Pi \leq 1, \\
& \Pi \geq \frac{\eta}{\eta^{\text {rev }}} \leq \frac{1}{2}, \quad \Pi \leq \frac{\eta}{\eta^{\text {rev }}} \geq \frac{1}{2} .
\end{aligned}
$$

The PCC may be a better coefficient than $\eta / \eta^{\text {rev }}$ for estimating real running machines. According to Eq. (71), the quotient $\eta / \eta^{\text {rev }}$ is too "optimistic", if $\eta / \eta^{\text {rev }} \geq 0.5$, and too "pessimistic", $\eta / \eta^{\text {rev }} \leq 0.5$.

\subsection{Novikov engine with Newtonian heat conduction}

In this section, we study a different subfamily of comparing processes all characterized by the same second simulation parameter $\lambda^{\mathrm{N}}$. Maximizing the power (42)

$$
P\left(T_{\mathrm{i}}, \lambda^{\mathrm{N}}\right)=\lambda^{\mathrm{N}} T_{\mathrm{L}}\left(1-\frac{T_{\mathrm{i}}}{T_{\mathrm{L}}}\right)\left(1-\frac{T_{\mathrm{H}}}{T_{\mathrm{i}}}\right)
$$

with respect to $T_{\mathrm{i}}$ yields an internal maximum for $T_{\mathrm{i}}^{2}=T_{\mathrm{L}} T_{\mathrm{H}}$, which results in the well-known Curzon-Ahlborn efficiency:

$$
\eta_{\mathrm{N} . \mathrm{N}}=\eta_{\mathrm{CA}}=1-\left(\frac{T_{\mathrm{L}}}{T_{\mathrm{H}}}\right)^{1 / 2} .
$$

One very interesting feature of this result as well as of that of the previous section is that the efficiency at maximum power does not depend on the value 
chosen for $\lambda$. This gives the result a much wider application than anticipated: in both cases, the efficiency obtained can be used as comparison measure for all members of the process family (14) and not only for the one with the particular $\lambda$ chosen for the simulating process.

\subsection{Novikov process with a Fourier heat leak}

As already mentioned in the section where the model was introduced, the existence of two first and of two second simulation parameters gives an additional degree of freedom. This degree of freedom makes it impossible to use the same technique in fixing the simulation parameter as in the previous sections. We thus proceed a little differently: We introduce a subfamily of processes that are characterized by a certain machine diagram depending on the variables $T_{\mathrm{i}}, \lambda$, and $\kappa$.

The reversibility condition

$$
-\frac{Q_{\mathrm{H}}-\Delta Q}{T_{\mathrm{i}}}-\frac{Q_{\mathrm{L}}+\Delta Q}{T_{\mathrm{L}}}=0
$$

allows determining $Q_{\mathrm{L}}$ as a function of $T_{\mathrm{i}}, Q_{\mathrm{H}}$, and $\Delta Q$ :

$$
Q_{\mathrm{L}}=-\frac{T_{\mathrm{L}}}{T_{\mathrm{i}}} Q_{\mathrm{H}}-\Delta Q\left(1-\frac{T_{\mathrm{L}}}{T_{\mathrm{i}}}\right)
$$

Inserting the defining equation (51)

$$
Q_{\mathrm{H}}=\lambda \tau\left(\frac{1}{T_{\mathrm{i}}}-\frac{1}{T_{\mathrm{H}}}\right), \quad \Delta Q=\kappa \tau\left(\frac{1}{T_{\mathrm{L}}}-\frac{1}{T_{\mathrm{i}}}\right)
$$

for both the second simulation parameters, we obtain the power

$$
P=\left(\frac{1}{T_{\mathrm{L}}}-\frac{1}{T_{\mathrm{i}}}\right)\left(\lambda\left(\frac{1}{T_{\mathrm{i}}}-\frac{1}{T_{\mathrm{H}}}\right)-\kappa\left(\frac{1}{T_{\mathrm{L}}}-\frac{1}{T_{\mathrm{i}}}\right)\right) .
$$

Its interpretation is easy: from $Q_{\mathrm{H}}(76)$ one subtracts the heat $\Delta Q$ bypassing the inner Carnot engine, multiplies it with the efficiency of the Carnot engine, and divides by $\tau$. Now the engine parameters $\lambda$ and $\kappa$ are again chosen from the original process. Within this process family, we look for the process with the highest power output. Differentiating Eq. (77) with respect to $T_{\mathrm{i}}$ leads to an optimal temperature 


$$
T_{\mathrm{i}}^{\mathrm{opt}}=\frac{2(\lambda+\kappa)}{\lambda\left(\frac{1}{T_{\mathrm{L}}}+\frac{1}{T_{\mathrm{H}}}\right)+2 \frac{\kappa}{T_{\mathrm{L}}}} .
$$

The efficiency at maximum power is

$$
\eta_{\mathrm{N} . \mathrm{FHL}}^{\mathrm{opt}}=\left(1-\frac{T_{\mathrm{L}}}{T_{\mathrm{H}}}\right) \frac{\lambda}{2 \lambda+4 \kappa}=\frac{1}{2} \eta_{\mathrm{rev}} \frac{1}{1+2 \frac{\kappa}{\lambda}}<\frac{1}{2} \eta_{\mathrm{rev}} .
$$

We see that the efficiency is always below the efficiency at maximum power for the Novikov engine with Fourier heat conduction due to the additional loss occurring in the heat leak.

\section{Discussion}

For a given 2-reservoir real running heat to power conversion process, we introduced four different endoreversible models for simulating the conversion process. This procedure is different from that found in the literature: here the idealized (in nature not running) endoreversible processes are directly connected to real running processes by simulating them. By this simulation, the machine parameters of the endoreversible models (the first simulation parameter "heat leak" and the second simulation parameter "heat conductivity") are determined by the real running process, and consequently, they cannot be chosen freely, as it is often assumed in the literature.

Fixing the machine parameters by the simulation procedure, we obtain a model-dependent family of comparative endoreversible processes, and we showed how these can be used as a basis for comparing efficiencies of dissipative thermodynamic processes. Apart from the Carnot engine with heat leak, the basis for the comparison were the efficiencies at maximum power of the respective model. This seems to be a reasonable basis, as usually heat to power conversion devices are intended to provide as much power as possible due to the investment made. Of course, in real power stations this is balanced with efficiencies obtained on an economical basis.

The efficiency comparison at maximum power yields different expressions for the efficiencies depending on the chosen model and on the temperature ratio $T_{\mathrm{L}} / T_{\mathrm{H}}$. This is due to the different structure (machine diagram) of the models. However, we note that for temperature ratios $T_{\mathrm{L}} / T_{\mathrm{H}}$ above 0.5 the difference is less than 10 percentage points apart from the Carnot efficiency. Thus one finds that whatever model is used, the suggested value for the efficiency is close to the proper range.

Interestingly, the efficiencies of the first three models discussed above do not depend on the special values chosen for the machine parameters. This is 
different for the last model, for which the values of the machine parameters will depend on the special considered process class $z$. The reason for that dependence is that there are more machine parameters than those that can be fixed by simulation. Generically, a real machine with its machine diagram is characterized by a subfamily of the process family $\mathscr{M}^{3}\left(T_{\mathrm{H}}, T_{\mathrm{L}}\right)$. Here one of its many process classes $\boldsymbol{z}$ is used to choose particular simulation parameters from which efficiency and power output now depend.

Nonetheless, it remains an open question which of the models might be more appropriate for the given original process. At the level of knowledge available about that process, such a question cannot be decided. One would need more information for instance about the major loss terms in the process under consideration, such that the structure of the chosen model captures the most essential loss terms. While this seems obvious, there remains nonetheless the fact that the Carnot efficiency used as a guide to possible efficiencies of heat to power conversion processes suffers from the same defects. Thus one cannot stress enough the point that the Carnot efficiency provides a bound to the efficiencies of real processes, but should not be mistaken as a good guide to the achievable values of a real process.

While our considerations here are confined to heat engines, the conceptual approach described above can be used as well for analyzing other thermodynamic processes such as distillation $[17,18]$, photovoltaics $[19,8]$, or chemically driven engines [20].

In the future, we thus need to undertake further research activities to define more sensible simulation models for irreversible processes that are connected to the main dissipative loss features of the process under consideration. We regard the question raised at the beginning of finite-time thermodynamics as still being open. The problem of constructing generic comparison processes by coupling them to properties of a real given process is yet to be solved in a more satisfactory fashion.

\section{Acknowledgements}

W.M. thanks Prof. Dr. H.H. Pucher, TU Berlin, Institut für Maschinenkonstruktion, for elucidating discussions on the scheme of a machine diagram (Figure 3). Financial support by the VISHAY Company, D-95085 Selb, Germany, is gratefully acknowledged.

\section{References}

[1] Muschik, W., Endoreversible thermodynamics: a tool for simulating discrete systems, in: Proceedings of Continuum Models and Discrete Systems (CMDS9), 
June 29-July 3, 1998, Istanbul, Turkey, Eds. E. Inan and K.Z. Markov, pp 47-55, World Scientific, Singapore, 1998.

[2] Elsner, N., Grundlagen der Technischen Thermodynamik, Kap. 8, AkademieVerlag, Berlin, 1973.

[3] Muschik, W., Aspects of Non-Equilibrium Thermodynamics, chap. 1.2, World Scientific, Singapore, 1990.

[4] Rubin, M.H., Optimal configuration of a class of irreversible heat engines I, Physical Review A, 19 (1979), 1272-1276.

[5] Andresen, B., Finite-time thermodynamics, in: Finite-Time Thermodynamics and Thermoeconomics, Advances in Thermodynamics 4, Eds. S. Sieniutycz and P. Salamon, p. 66, Taylor and Francis, New York, 1990.

[6] Hoffmann, K.H., Burzler, J.M., Schubert, S., Endoreversible thermodynamics, J. Non-Equilib. Thermodyn., 22 (1997), 311-355.

[7] Hoffmann, K.H., Burzler, J., Fischer, A., Schaller, M., Schubert, S., Optimal process paths for endoreversible systems, J. Non-Equilib. Thermodyn., 28 (2003), 233-268.

[8] de Vos, A., Endoreversible Thermodynamics of Solar Energy Conversion, Oxford University Press, Oxford, 1992.

[9] Amelkin, S.A., Andresen, B., Burzler, J.M., Hoffmann, K.H., Tsirlin, A.M., Maximum power processes for multi-source endoreversible heat engines, J. Phys. D, 37 (2004), 1400-1404.

[10] Amelkin, S.A., Andresen, B., Burzler, J.M., Hoffmann, K.H., Tsirlin, A.M., Thermomechanical systems with several heat reservoirs: maximum power processes, J. Non-Equilib. Thermodyn., 30 (2005), 67-80.

[11] Gordon, J.M., Huleihil, M., General performance characteristics of real heat engines, J. Appl. Phys., 72 (1992), 829-837.

[12] Novikov, I.I., The efficiency of atomic power stations, J. Nuclear Energy II, 7 (1958), 125-128 (translated from Atomnaya Energiya, 3 (1957), 409).

[13] de Vos, A., Efficiency of some heat engines at maximum-power conditions, Am. J. Phys., 53 (1985), 570-573.

[14] Fischer, A., Hoffmann, K.H., Can a quantitative simulation of an Otto engine be accurately rendered by a simple Novikov model with heat leak?, J. NonEquilib. Thermodyn., 29 (2004), 9-28.

[15] Muschik, W., Aspects of Non-Equilibrium Thermodynamics, chap. 3, World Scientific, Singapore, 1990.

[16] Kestin, J., A Course in Thermodynamics, Vol. I, Hemisphere, Washington, 1979.

[17] Schaller, M., Hoffmann, K.H., Rivero, R., Andresen, B., Salamon, P., The influence of heat transfer irreversibilities on the optimal performance of diabatic distillation columns, J. Non-Equilib. Thermodyn., 27 (2002), 257-256.

[18] Koeijer, G.M., Kjelstrup, S., Salamon, P., Siragusa, G., Schaller, M., Hoffmann, K.H., Comparison of entropy production rate minimization methods for binary diabatic distillation, Ind. Eng. Chem. Res., 41 (2002), 5826-5834.

[19] Landsberg, P.T., Baruch, P., The thermodynamics of the conversion of radiation energy for photovoltaic, J. Phys. A, 22 (1989), 1911-1926.

[20] Badescu, V., Andresen, B., Probabilistic finite time thermodynamics: a chemical driven engine, J. Non-Equilib. Thermodyn., 21 (1996), 291-306.

Paper received: 2005-12-23

Paper accepted: 2006-02-15 
Bereitgestellt von | Technische Universität Berlin 